\title{
The identification problem solution of the ventilation system model based on static pressure
}

\author{
Dmitriy.V. Boykov ${ }^{1,2, ~ *}$, Aleksandr A. Dekterev ${ }^{1,2}$ \\ ${ }^{1}$ Institute of Thermophysics SB RAS, Novosibirsk \\ ${ }^{2}$ Siberian Federal University, Krasnoyarsk
}

\begin{abstract}
A pressure method to solve the problem of flow distribution in a pipeline system based on the static pressure use was proposed in the study. A modification of the identification method to search for the local resistance coefficients of the ventilation system sites was provided. Static pressure and flow rate as identifying values were used in the developed approach. The applicability of the identification method for local resistance of the gas removal system to be found was shown.
\end{abstract}

\section{Introduction}

When analyzing the systems operation supporting the gas flow, different research methods can be used [1,2]: the theory of hydraulic circuits [3], 3d modeling methods [4,5], test measurements of real-life objects [6]. In this case, the results obtained by one method in other methods are certain to be used [1]. An example is the identification problem of a network model according to test measurements of the current ventilation system [6]. Currently, there is a varied mathematical apparatus aimed at solving such problems in pipeline systems $[1,3,7-$ 12]. However, the use of these methods for solving the flow distribution and identification problems in ventilation systems seems to be difficult. They are associated with the use of total pressure in solving problems of flow distribution and identification in the pipeline system [13].

Consider an example of the off gas for an electrolysis production in the aluminum plant (Fig. 1). It is designed for electrolysis gases to be discharged from electrolysis baths to gas purification. The system within one potroom is divided into 4 sets with 20 electrolysis baths in each. In the given system, uncontrolled parasitic air inflows into the off gas through fabrication holes (measurement hatches, dielectric loadings) is observed.

The total value of such parasitic air inflows is up to one third of the total system flow rate at the system exit [6]. For a small diameter hole (measurement hatches) on the pipe wall, this parasitic flow - rate has been determined by the following relation [14]:

\footnotetext{
*Corresponding author: dimkadim@yandex.ru
} 


$$
q_{l}=C_{e l} \frac{\pi d_{i}^{2}}{4} \sqrt{\frac{2 \Delta p_{i}}{\rho}}
$$

where $C_{e l}$ is an entrainment ratio, $d_{l}$ is a hole diameter $(\mathrm{m}), \Delta p$ is a difference of static pressure in the pipe and in the environment $(\mathrm{Pa}), q_{l}$ is a flow - rate $(\mathrm{nm} 3 / \mathrm{h})$. Similar relations are applied to other parasitic air inflows associated with pipe walls leakage. Pressure losses in all other sections are described by the Bernoulli equation $[14,15]$ :

$$
\Delta\left(p_{i}+\left|\rho v^{2} / 2\right|_{i}+\rho g z_{i}\right)=\left(\xi_{l}+\frac{\lambda_{l} \cdot L_{l}}{d_{l}}\right) \frac{\left|q_{l}\right| \cdot q_{l}}{2 \rho s_{l}}-h_{l},
$$

where $p_{i}$ is a static pressure $(\mathrm{Pa}),\left|\rho v^{2} / 2\right|_{i}$ is a dynamic pressure $(\mathrm{Pa}), \rho g z_{i}$ is a geometric pressure $(\mathrm{Pa}), v$ is a velocity $(\mathrm{m} / \mathrm{s}), \lambda_{l}, \xi_{l}$ is a linear and local resistance coefficient, $d_{l}$ is a hydraulic diameter $(\mathrm{m}), L_{l}$ is a section length $(\mathrm{m}), h_{l}$ is a fixed head $(\mathrm{Pa})$. The dynamic pressure is comparable to the static pressure, with the geometric pressure being low, compared to plumbing and heat supply systems in ventilation systems.

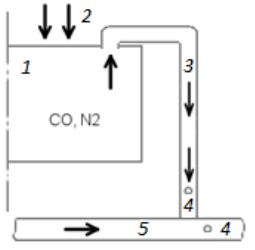

a

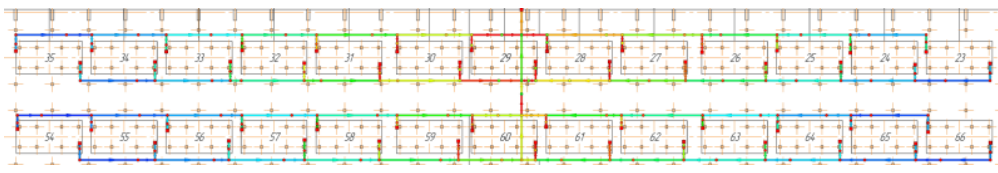

b

Fig. 1. The system of electrolysis production gas flue.

a) electrolysis bath connection

1 -electrolysis bath; 2 - air inflows; 3 - discharge; 4 - metering points;

5 - collecting gas duct

b) network

The measurement technique in the ventilation system [16,17] allows one to determine the static pressure and flow - rate values at the measurement points, in some points only one of these values being available. The proximity of structural elements (local resistances) followed by an uneven flow make it difficult to measure the flow - rate which is unlikely to be measured because of the measurement hatches size. When measuring the system (Fig. 1), it was impossible to measure the flow-rate at $10 \%$ of the measurement points.

In this case the problem of identification is to determine the local resistance coefficients of network sections, using the known static pressure and flow rate values. Identification coefficient of local resistance for some groups of identifiable branches are suggested to be the same due to availability of structurally identical network elements in their constructive and operating mode [1]. An example would be a section including an electrolysis bath and a discharge into a longitudinal gas duct (1-3 in Fig. 1).

Enhancement of the currently available methods for calculating the flow distribution $[3,7,8]$ and solving the identification problem [1,9-11], with the static pressure used when calculating, has been required.

\section{Research objective}

As a mathematical model for describing fluid flow in a network, a steady flow model of incompressible fluid has been taken. To represent the pipeline system, a directed graph is used [18], and the coupling matrix is as follows: 


$$
D_{i l}= \begin{cases}1, & \text { if } l \in O_{i}, \\ -1, & \text { if } l \in I_{i}, \\ 0 & \text { otherwise }\end{cases}
$$

Here $l \in O_{i}$ is the set of pipes (branches) coming from the $i$-th node; $l \in I_{i}$ is the set of pipes included in the $i$-th node. The flow distribution problem in the network can be reduced to a combination of the law of conservation of mass in the node (4) and the resistance law in the branch (pipe) (5):

$$
\begin{gathered}
\sum_{l \in U_{j}} D_{j l} q_{l}=0 \\
\sum_{i \in N} D_{i l} p_{i}=h_{l}\left(q_{l}\right)
\end{gathered}
$$

Here $\mathrm{N}$ is the set of all network nodes; $\mathrm{U}$ is the set of all network branches; $h_{l}\left(q_{l}\right)$ is the pressure loss $(\mathrm{Pa})$. Also, the set of nodes and the set of branches were naturally divided into two subsets: the boundary $\mathrm{N}_{\mathrm{bdr}}$ and the calculated $\mathrm{N}_{\mathrm{clc}}$ nodes, the boundary $\mathrm{U}_{\mathrm{bdr}}$, and the calculated $U_{\text {clc }}$ branches [1]. The pressure is fixed in the boundary nodes; the flow rate is in the boundary branches.

The Bernoulli equation (2) is reduced to the following form $\Delta p=\left(\xi_{l}+\frac{\lambda_{l} \cdot L_{l}}{d_{l}}\right) \frac{|v| \cdot v}{2}-$ $\left(h_{l}+\rho_{l} g \Delta z_{i}+\Delta\left[\frac{\rho v^{2}}{2}\right]_{i}\right)$. This allows one to solve the flow distribution problem in the hydraulic circuit, taking into account, within the same system of equations (4), (5), parasitic air inflows (1) and ordinary sections (2).

The dynamic pressure for the boundary nodes is 0 , for all others it is equal to the average flow rate value of the dynamic pressure of the flows going into this node:

$$
\left|\frac{\rho v^{2}}{2}\right|_{i}=\sum_{l \in I_{i}(q)} \frac{q_{l}^{2}}{2 \cdot s_{l} \cdot \rho}\left|q_{l}\right| / \sum_{l \in I_{i}(q)}\left|q_{l}\right|
$$

The identification problem is set as follows: Determine the minimum values of local resistance coefficients $\left\|\xi_{\mathrm{m}}\right\|_{2} \rightarrow$ min for identifiable branches and groups of identifiable branches satisfying the condition for minimum, distinguishing the calculated and experimental values $\|M\|_{2} \rightarrow \min$ of the static pressure $M_{I}=P_{I}-p_{I}, I \in c N$ and the flow - rate $M_{I}=\left(\frac{\partial h_{I}}{\partial q_{I}}\right)$.

$\left(Q_{I}-q_{I}\right), I \in c E$ at the control points $c N, c E$ under the system of equations (4), (5). Here $P_{I}, p_{I}$ are measured and calculated static pressures at control points $(c N), Q_{I}, q_{I}$ are the measured and calculated flow rate at the control points $(c E)$. The network topology, the geometrical dimensions of all sections are given. The condition for minimum of the required local resistances results from the maximum likelihood estimate $[7,19]$.

\subsection{Pressure method}

To solve the problem of flow distribution with respect to static pressure, the pressure method was used $[3,7]$. The value and pressure are found by an iterative cycle for pressure correction:

$$
p_{i}^{K+1}=p_{i}^{K}+\delta p_{i}
$$




$$
\begin{gathered}
\sum_{i \in N} \Delta_{i j}(a) \delta p_{i}=-\sum_{l \in U_{j}} D_{i l} q_{l}, j \in N_{c l c} \\
\delta p_{j}=0, j \in N_{b d r} \\
\Delta_{i i}(a)=\sum_{l \in U_{l}} a_{l}, \quad i \in N_{c l c} \\
\Delta_{i j}(a)=-\sum_{l \in U_{l}} a_{l}, \quad i \in N_{c l c}, j \in N, i \neq j \\
a_{l}=\left\{\begin{array}{l}
\left(\frac{\partial h_{l}}{\partial q_{l}}\right)^{-1}, l \in U_{c l c} \\
0, l \in U_{b d r}
\end{array}\right.
\end{gathered}
$$

To provide convergence, the dynamic pressure is recalculated in the external cycle, after several iterations with the SLAE solution (8). The required norm of flow-rate residual at the nodes when the internal iteration cycle is completed should be established no more than $10 \%$ of this value after recalculating the dynamic pressure (6) in the step of the external iteration cycle. This condition is the only significant difference from the conventional pressure method.

\subsection{Identification method of the ventilation system on static pressures and flow rates}

The identification problem is solved by an iteration cycle [1]:

$$
\xi^{K+1}=\xi^{K}+d \xi
$$

Then the SLAE relating the change in the adjusted local resistance to the resulting pressure change in the nodes is as follows:

$$
\begin{gathered}
\sum_{i \in N} \Delta_{i j}(a) \frac{d p_{i}}{d \xi_{m}}=\sum_{l \in U} \nabla_{i l}\left(\frac{\partial h_{l}}{\partial x_{l}}\right)^{-1}\left(\frac{\partial h_{l}}{\partial \xi_{m}}\right), j \in N_{\text {inner }}, m \in U_{\text {ident }} \\
\frac{d p_{i}}{d \xi_{m}}=0, \quad i \in N_{b d r}
\end{gathered}
$$

Where $d p_{i}^{m} / d \xi_{m}$ is the ratio of the static pressure change at the node $d p_{i}$ to the corresponding change in the identifiable value $d \xi_{m}, m \in U_{\text {ident }}$ is the set of identifiable objects.

The right side of SLAE (10) can be represented as:

$$
\left(\frac{\partial h_{l}}{\partial q_{l}}\right)^{-1}\left(\frac{\partial h_{l}}{\partial \xi_{m}}\right) d \xi_{m}=\left|q_{l}\right| / 2\left(\frac{\lambda_{l} l_{l}}{d_{l}}+\xi_{m}\right) d \xi_{m}
$$

It is not difficult to demonstrate that the resulting pressure change for the sum of the various impacts is equal to the sum of the pressure changes of these effects. Thus, the new values of local resistance are likely to be calculated by solving the SLAE:

$$
\begin{gathered}
\left\|A_{M I} \xi_{M}^{K+1}-\left(M_{I}+A_{M I} \xi_{M}^{K}\right)\right\|_{2} \rightarrow \min \\
A_{M I}=\frac{d p_{I}^{m}}{d \xi_{m}}, M_{I}=P_{I}-p_{I}, m \in U_{\text {ident }}, I \in c N \\
A_{M I}=\sum_{i \in N} \nabla_{i I} \frac{d p_{i}^{m}}{d \xi_{m}}-\frac{\partial h_{m}}{\partial \xi_{m}}, M_{I}=\left(\frac{\partial h_{I}}{\partial q_{I}}\right) \cdot\left(Q_{I}-q_{I}\right), m \in U_{\text {ident }}, I \in c E
\end{gathered}
$$

Here, $\xi_{M}^{K+1}, \xi_{M}^{K}$ is the vector of identifiable local resistances of the current and past iteration. To solve the SLAE (12), the least squares method is used [20-21]. 
Metering points that coincide with the boundary network elements are separately taken into account; such points are directly given as the corresponding boundary conditions in the equations system (4), (5).

\section{Test}

The problem of identification of the off gas system was solved. To achieve that, 65 pressure and 71 flow rate measurements were made. local resistance coefficients of 550 sections were evaluated, the problem was reduced to obtaining 14 independent and 3 group local resistance coefficients [1].

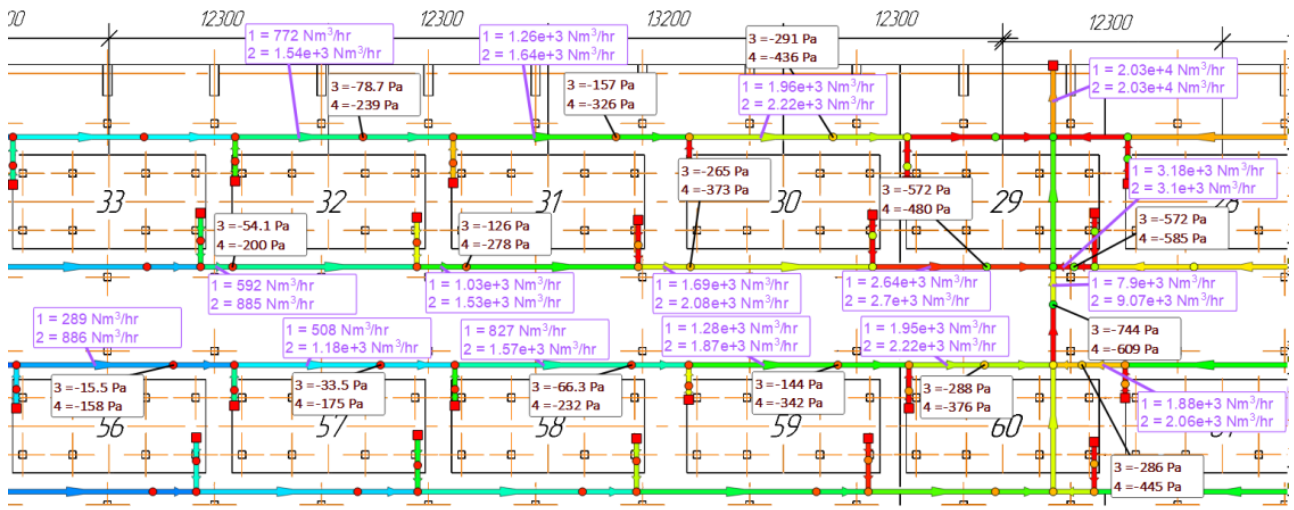

a

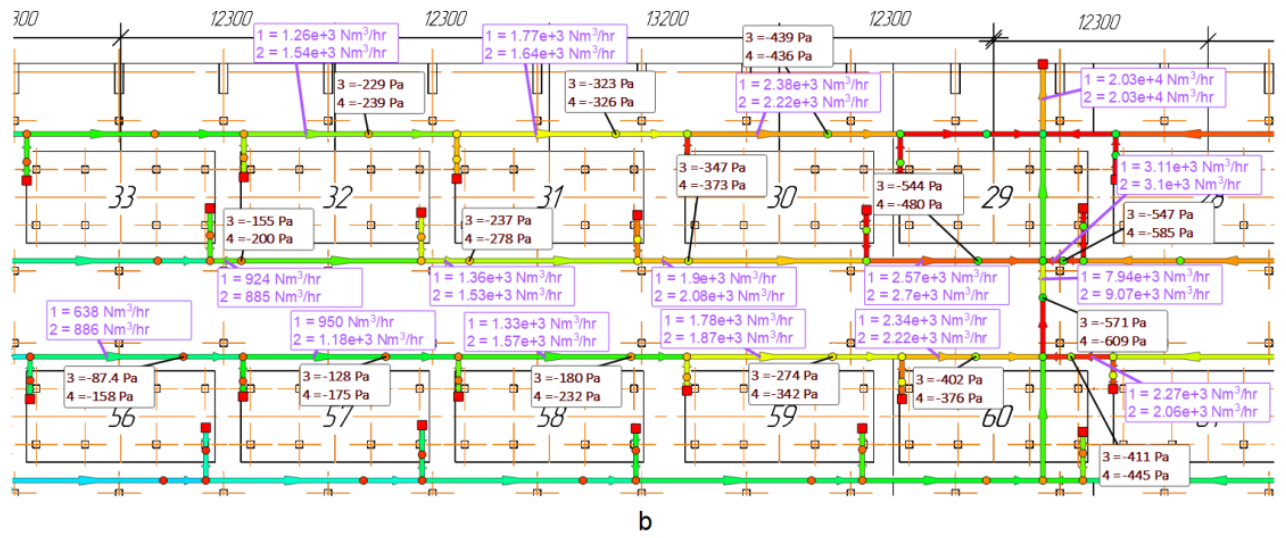

Fig. 2. Comparison of the experiment with the calculation, electrolyzer 33-29, 56-60.

a) before identification

b) after identification

1- Flow rate calculated by the network model; 2 - flow measurement; 3 - static pressure calculated by the network model; 4 - pressure measurements

The calculation results before and after identification has been shown in Fig.2. The identification reduced the 12 norm of the difference between experimental and calculated pressure values by a factor of 2.5. After identifying, the pressure and flow rates values according to the calculation, register perfectly with the experimental data. 


\section{Conclusion}

The modification for the static pressure of the nodal pressure method has been proposed, so has the identification method for local resistances of network elements by static pressures and flow rates at test points. According to the experiment the given method was used to adapt the system of gas ducts for the electrolysis production.

This work was carried out under state contract with IT SB RAS (AAAA-A17-117030910025-7).

\section{References}

1. D. Boykov, A. Dekterev, E3SWeb Conf, 39, Article Number 01001 (2018)

2. D. Boykov, S. Filimonov, A. Dekterev, Pipeline energy systems. Mathematical and computer technologies of intellectualization, 138 (2017)

3. A.P.Merenkov.V.Ya.Hasilev, Theory of hydraulic circuits (1985)

4. J.H. Ferziger, M. Peric, Computational Methods for Fluid Dynamics (2002)

5. A.A. Dekterev, K.Yu. Litvintsev, A.A. Gavrilov, E.B. Kharlamov, S.A. Filimonov Journal of Siberian Federal University. Engineering \& Technologies, 10(4) (2017)

6. S. Shakhrai, E. Rzhechitsky, V. Kondratiev, A. Dekterev, D. Boykov, Collection of reports of the XIV Intern. Conf.-Exhibition Aluminum of Siberia, 408 (2008)

7. N. N. Novitskii Thermal engineering, 60(14), 1051 (2013)

8. E. Todini, Bollettino degli Ingegneri della Toscana, 11, 11 (1979)

9. S. Epifanov, N. Novitsky, Pipeline energy systems. Methods of mathematical modeling and optimization, 27 (2007)

10. N. Novitsky, Estimation of parameters of hydraulic circuits (1998)

11. Z. Y. Wu, C. Clark, Water Resources Management, 23(1), 117 (2009)

12. Bragalli, C., Fortini, M., Todini, E. Water Resources Management, 30(11), 3689 (2016)

13. J. R. Travis, J. W. Spore, P. Royl, and other, GASFLOW-II: A Computational Fluid Dynamics Code for Gases Aerosols, and Combustion, 1 (2011)

14. D.Miller, Internal flow systems(1996)

15. I. Idelchik, Handbook of hydraulic resistance (1992).

16. GOST 17.2.4.06-90, Protection of Nature. Atmosphere. Methods for determining the speed and gas and dust flow rates from stationary sources of pollution

17. Flow pipes of NIIOGAZ and Pitot modifications. Operation manual 3.820.000 ORM

18. R. Wilson. Introduction to graph theory (1996)

19. Y. Bard, Non-Linear Parameter Estimation (1974)

20. Benoît Jacob, Gaël Guennebaud, Eigen 3 documentation

21. G. H. Golub, Ch. F. van Loan, Matrix Computations. (1996) 